\title{
FRACTAL NEWTON BASINS
}

\author{
M. L. SAHARI AND I. DJELLIT \\ Received 3 June 2005; Accepted 14 August 2005
}

The dynamics of complex cubic polynomials have been studied extensively in the recent years. The main interest in this work is to focus on the Julia sets in the dynamical plane, and then is consecrated to the study of several topics in more detail. Newton's method is considered since it is the main tool for finding solutions to equations, which leads to some fantastic images when it is applied to complex functions and gives rise to a chaotic sequence.

Copyright (C) 2006 M. L. Sahari and I. Djellit. This is an open access article distributed under the Creative Commons Attribution License, which permits unrestricted use, distribution, and reproduction in any medium, provided the original work is properly cited.

\section{Introduction}

Isaac Newton discovered what we now call Newton's method around 1670. Although Newton's method is an old application of calculus, it was discovered relatively recently that extending it to the complex plane leads to a very interesting fractal pattern.

We have focused on complex analysis, that is, studying functions holomorphic on a domain in the complex plane or holomorphic mappings. It is a very exciting field, in which many new phenomena wait to be discovered (and have been discovered). It is very closely linked with fractal geometry, as the basins of attraction for Newton's method have fractal character in many instances. We are interested in geometric problems, for example, the boundary behavior of conformal mappings. In mathematics, dynamical systems have become popular for another reason: beautiful pictures. Principally, we have focused on Julia sets and the iteration of rational functions. The Newton's method is one of the most important iterative algorithms for finding the zeroes of a polynomial or, more generally, a function meromorphic on the complex plane.

We first discuss some general properties of the method for rational functions with complex roots, including convergence criteria and rates of convergence, periodic points and basins of attractions of the roots. Next, we present results of Newton's method for one-dimensional and two-dimensional cases. Applying Newton's method to cubic function, whose roots are known as the 3 th roots of unity, we show that the boundaries of the 
basins of attraction of each root are typically fractal in nature, and that they correspond to Julia sets in the complex plane. We conclude with a discussion of the implications of the fractal structure on the utility of Newton's method as a root-finding algorithm.

A Newton basin is just the set of initial guesses that lead to one solution or root. What is interesting is that Newton basins are fractals! and have beautifully intricate boundaries. In fact, they are kinds of Julia sets.

We refer to the surveys [2-4] for the general view of the dynamics of complex maps. We will use some concepts and facts of this theory without extra explanations.

Recall that one of the most important iteration schemes is Newton's method for finding the roots of algebraic equations equivalently, solutions to equations of the form

$$
z_{k+1}=\mathcal{N}\left(z_{k}\right) .
$$

Not only is the method easy to comprehend, it is a very efficient way and very helpful tool for finding roots as long as the function converges. In general, Newton's method works impressively fast (with quadratic convergence). But it requires a good initial guess, which normally needs to be close to one of the roots. Otherwise, strange things may happen. Historically the survey relative to basins of attraction of a map generated by the Newton's algorithm related to the quadratic equation resolution with complex variable has been studied initially by Schroeder (1871). The cubic case

$$
a z^{3}+b z^{2}+c z+d=0 ; \quad(a, b, c, d) \in \mathbb{R}^{4}
$$

developed by Cayley in 1879 [2]. Specifically, Cayley wished to characterize the global basins of attraction for each root, more precisely, these basins of attraction are the initial conditions that Newton's method carries to each root. This task of identifying such basins for complex polynomials is known as "Cayley's problem." The works of Cayley have been reconsidered by Gaston Julia, who creates the Julia theory.

In this work we examine the chaotic behavior of a function of cubic type

$$
z^{3}-1=0
$$

First, we study the geometry of the Julia set relative to the map (1.1) and we calculate its fractal dimension (Figure 1.1).

By construction, the Julia set is the set of points for which Newton's method fails to find a root.

\section{Notations, definitions and fundamental results}

We denote $\mathbb{C}$ the complex plane; we define the Riemann sphere $\overline{\mathbb{C}}$ as $(\overline{\mathbb{C}}=\mathbb{C} \cup\{\infty\})$.

Let us consider the complex map

$$
z_{k+1}=g\left(z_{k}\right)
$$

Definition 2.1. We define the set of wandering points by

$$
D_{g}(\infty)=\left\{z: g^{k}(z) \longrightarrow \infty, k \longrightarrow \infty\right\}
$$




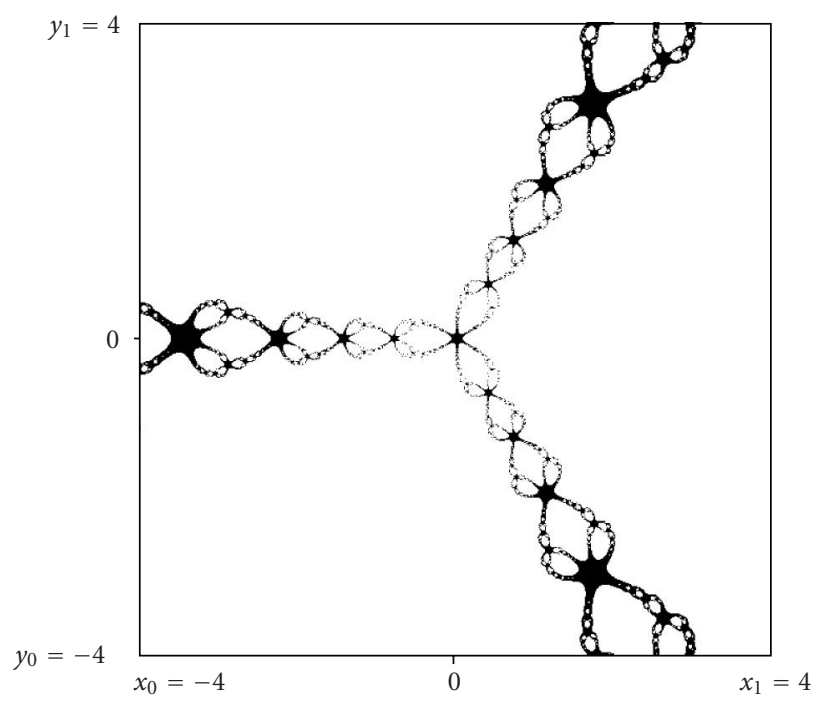

Figure 1.1. Basin boundaries are pictured of the three roots of (1.3).

Definition 2.2. The Fatou set $\mathscr{F}(g)$ of nonwandering points is defined by

$$
\mathscr{F}(g)=\mathbb{C} \backslash D_{g}(\infty) .
$$

Definition 2.3. The sets $D_{g}(\infty)$ and $\mathscr{F}(g)$ have a common boundary which is the Julia set $\mathscr{F}(g)$, then

$$
\mathscr{f}(f)=\partial\left(D_{g}(\infty)\right)=\partial(\mathscr{F}(g)) .
$$

Definition 2.4. The basin of attraction $\mathscr{A}(\omega)$ of an attractive fixed point $\omega$, associated with a function $g$ is

$$
\mathscr{A}(\omega)=\left\{z \in C: g^{k}(z) \underset{k \rightarrow \infty}{\longrightarrow} \omega\right\}
$$

Definition 2.5. A point $\alpha$ is a fixed point of $g$ if $g(\alpha)=\alpha$, and $p$-periodic if $g^{p}(\alpha)=\alpha$, for all $p \in \mathbb{N}$. The set $\left\{g^{k}(\alpha)\right\}_{k=0, \ldots, p-1}$ is called orbit or cycle.

\section{The Newton's method}

Isaac Newton devised an algebraic procedure for a rapidly convergent sequence of successive approximations to a root of the equation $f(z)=0, z \in \mathbb{C}$. Newton's derivation is equivalent to the modern iterative procedure described by the equation.

Solving the polynomial equation with real coefficients in $\mathbb{C}$ given here by

$$
f(z)=0, \quad z \in \mathbb{C} .
$$

Definition 3.1. Let $f$ be a polynomial and let $z_{k}$ be the $k$ th iterate under Newton's method of the point $z_{0}$. Then we say that $z_{0}$ is an approximate zero of $f$. 
The basic idea is to construct a recurrent sequence $\left\{z_{k}\right\}_{k=0,2, \ldots}$ such as $f\left(z_{k}\right) \rightarrow 0$. Assume that the image $z_{k}$ is known; (3.1) linearized in $z_{k}$ gives

$$
f(z)=f\left(z_{k}\right)+\left(z-z_{k}\right) f^{\prime}\left(z_{k}\right)
$$

the solution is the following image $z_{k+1}$, then

$$
z_{k+1}=z_{k}-\frac{f\left(z_{k}\right)}{f^{\prime}\left(z_{k}\right)}
$$

if

$$
f(z)=z^{n}-1, \quad n \in \mathbb{N}
$$

replacing in (3.3) and taking into account that

$$
z=x+i y
$$

we obtain the well-known equation as Newton's sequence

$$
(n-1) z_{k}^{n}-n z_{k+1} z_{k}^{n-1}+1=0 .
$$

\section{Convergence property}

The Newton's algorithm converges globally if for all $z \in \mathbb{C}$ a subsequence of $\left\{z_{k}\right\}_{k \in \mathbb{N}^{*}}$ which tends to $z^{*}$ such that $\left|f\left(z^{*}\right)\right| \simeq 0$. We test the efficiency of the algorithm by the exam maps that it generated. The notion of convergence speed permits to qualify the asymptotic behavior of a mapping.

4.1. Order of convergence. We are interested by the following rate

$$
q_{k}=\frac{\left|z_{k+1}-z^{*}\right|}{\left|z_{k}-z^{*}\right|} .
$$

(i) If $\lim \sup q_{k}=1$; the convergence is sublinear.

(ii) If $\lim \sup q_{k}=\alpha<1$; the convergence is then linear and $\alpha$ is the associated ratio of convergence.

(iii) If $\lim q_{k}=0$; the convergence is superlinear.

(iv) If $\exists \gamma>1$ such that $\lim \sup \left(\left|z_{k+1}-z^{*}\right| /\left|z_{k}-z^{*}\right| \gamma\right)=M<+\infty$; the convergence is superlinear of order $\gamma$. In particular for $\gamma=2$ we have a quadratic convergence, and $\left|z_{k}-z^{*}\right|^{2}$ is called the residual measure.

Theorem of Newton-Kantorovich [1]. Suppose that $f$ is twice continuously differentiable and call $z^{*}$ a zero of $f$ then if $f^{\prime}\left(z^{*}\right) \neq 0$ and $z_{0}$ is sufficiently close of $z^{*}$. The map converges to $z^{*}$ and we have a quadratic convergence.

The final result that we know on the importance of linking fractal geometry with numerical analysis is probably: "the global convergence pattern of Newton's method is predictable and consists of few (four) convergence states," related to the residual measure. At least for complex polynomials, we have this description. 
(i) Stationary residual with constant magnitude (usually 1-the exact magnitude depends on the distance between root of the function and relevant root of the first derivative).

(ii) A sharp increase in residual when the iterates reach a singularity (root of the first derivative). Assuming ultimate convergence, this has to be followed by the next state.

(iii) Linear convergence with a predictable rate $(n /(n-1)$ for polynomials of degree $n)$. This is the only type of convergence possible once the residual is larger than the stationary value. (For residuals slightly larger than the stationary value, linearity is only an approximation.)

(iv) Quadratic convergence once the iterates are sufficiently close to the root (the residual is smaller than the stationary value) — the well-known Newton-Kantorovich convergence result.

\section{Application on the cubic case "Cayley problem"}

In 1879, Cayley asked the following question: given an initial input $z_{0}$, to which root will Newton's method converge. The answer was only fully understood recently and leads to a beautiful fractal pattern. The other good thing about knowing the structure of the underlying Newton fractal is that we can tell which regions are bad for a starting guess (namely, the ones that have singular-Julia-points in them).

Next, we discuss Newton's method applied to the simple cubic equation

$$
f(z)=z^{3}-1
$$

The Newton's equation is expressed in the form

$$
2 z_{k}^{3}-3 z_{k+1} z_{k}^{2}+1=0
$$

more explicitly

$$
z_{k+1}=\mathcal{N}\left(z_{k}\right)=\frac{2 z_{k}^{3}+1}{3 z_{k}^{2}} .
$$

We note by $z_{+}=x_{+}+i y_{+}$and $z=x+i y$ instead of $z_{k+1}=x_{k+1}+i y_{k+1}$ and $z_{k}=x_{k}+i y_{k}$. If we replace in (5.3), we separate the real part of the imaginary part and after a simple calculation, we have

$$
\begin{gathered}
x_{+}=\frac{2 x^{5}+4 x^{3} y^{2}+2 x y^{4}+x^{2}-y^{2}}{3\left(x^{2}+y^{2}\right)^{2}}, \\
y_{+}=\frac{2 y\left[\left(x^{2}+y^{2}\right)^{2}-x\right]}{3\left(x^{2}+y^{2}\right)^{2}},
\end{gathered}
$$

which is simply the projection of the formula (5.3) on the real and imaginary axes.

5.1. Study of the one-dimensional Newton map. Consider first the restriction of Newton's algorithm in the one-dimensional space, we now specialize to the real case and it 
Table 5.1. Newton algorithm.

\begin{tabular}{lccccc}
\hline$x_{0}$ & -50.00 & -0.0001 & -1002.754 & 01.50 & 100.0 \\
$k_{\max }$ & 15 & 48 & - & 5 & 15 \\
\hline
\end{tabular}

suffices to replace in (5.3) $z_{+}$and $z$ by $x_{+}$and $x$ then

$$
x_{+}=\frac{2 x^{3}+1}{3 x^{2}}
$$

In the following Table 5.1, one summarized results of the algorithm of Newton with different initial points $\left(k_{\max }\right.$ represents the maximal number of iterations to reach the solution $\left.x^{*}=1.0\right)$.

It is clear (from (5.5)) that the algorithm fails only for $x=0$, then 0 belongs to Julia set as well as all $x$ such that $x \rightarrow 0$. Now the idea to construct the Julia set is to take images of the point at the origin by the inverse algorithm of Newton. Let us come back to (5.5) to solve this last with regard to $x$, we obtain that $x<1$ there is a unique solution, which is

$$
x=\frac{\left(-2+x_{+}^{3}+2\left(\sqrt{-x_{+}^{3}+1}\right)\right)^{2 / 3}+x_{+}^{2}}{2\left(-2+x_{+}^{3}+2\left(\sqrt{-x_{+}^{3}+1}\right)\right)^{1 / 3}}+\frac{x_{+}}{2},
$$

where the inverse formula of Newton's algorithm. Then we can construct the Julia set (see Figure 5.1) as union of points $\left\{\chi_{k}\right\}_{k=0,1,2, \ldots}$ defined by

$$
\begin{gathered}
\chi_{0}=0 \\
\chi_{1}=-\frac{1}{\sqrt[3]{2}} \\
\chi_{k+1}=\frac{\sqrt[3]{-2+\chi_{k}^{3}+2 \sqrt{-\chi_{k}^{3}+1}}+\chi_{k}^{2}}{2{\sqrt[3]{-2+\chi_{k}^{3}+2 \sqrt{-\chi_{k}^{3}+1}}}^{2}}+\frac{\chi_{k}}{2} .
\end{gathered}
$$

The map (5.7) does not admit a fixed point because we can verify that for $u<1$ we have

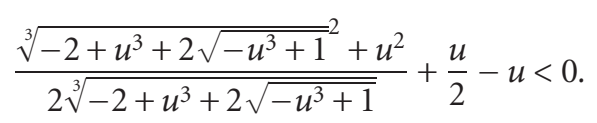

We remark that this last inequality implies the decrease of the mapping defined in (5.24) and that $\left\{\chi_{k}\right\}_{k=0,1,2, \ldots} \subset \mathbb{R}_{-}$. Let us examine now the behavior of the map $\left\{\chi_{k}\right\}$ at the infinity while calculating the quotient

$$
\frac{\chi_{k+1}}{\chi_{k}}=\frac{\Phi\left(\chi_{k}\right)^{2}+\chi_{k} \Phi\left(\chi_{k}\right)+\chi_{k}^{2}}{2 \chi_{k} \Phi\left(\chi_{k}\right)}=\frac{1}{2}\left(\Gamma+\frac{1}{\Gamma}\right)+\frac{1}{2} \text {. }
$$


M. L. Sahari and I. Djellit 7

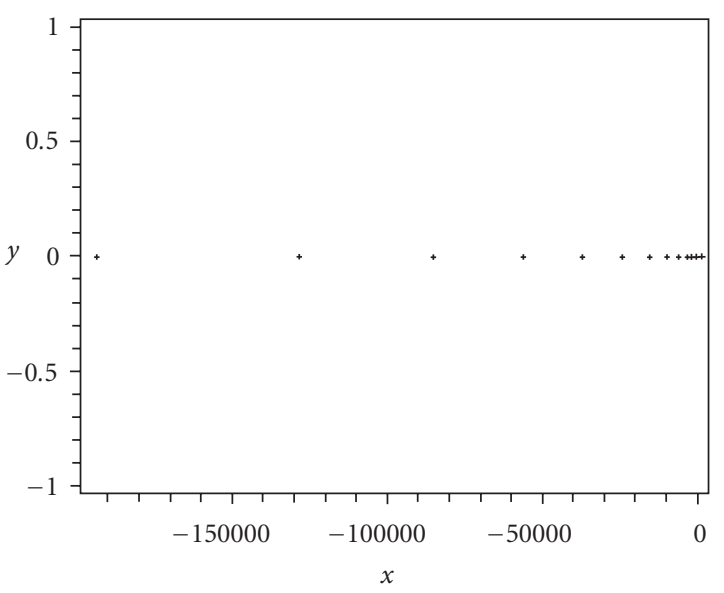

Figure 5.1. The Julia set in $\mathbb{R}$.

Where

$$
\begin{gathered}
\Phi(x)=\sqrt[3]{-2+x^{3}+2 \sqrt{1-x^{3}}} \\
\Gamma=\frac{\Phi\left(\chi_{k}\right)}{\chi_{k}} .
\end{gathered}
$$

Solving the extrema points

$$
\min _{\Gamma>0}\left(\Gamma+\frac{1}{\Gamma}\right)
$$

we verify that

$$
\frac{\chi_{k+1}}{\chi_{k}}>\frac{3}{2}
$$

and because $\chi_{k+1} / \chi_{k}>0$ and $\chi_{k}<0$ for all $k \geq 0$ then

$$
\lim _{k \rightarrow+\infty} \chi_{k}=-\infty
$$

the numerical tests (Table 5.2) validate the result (5.13).

If we put $l_{j}=\chi_{j+1}-\chi_{j} ; j=0,1,2, \ldots$ we remark that $l_{j+1} / l_{j}=3 / 2$ (see Figure 5.2 ) and then we have

$$
\chi_{j}=\frac{1}{\sqrt[3]{2}}\left(1-\sum_{i=0}^{i=j}\left(\frac{3}{2}\right)^{i}\right), \quad j=0,1,2, \ldots
$$


Table 5.2. Algorithm of Newton and its inverse.

\begin{tabular}{rcc}
\hline$k$ & Newton algorithm & Inverse Newton algorithm \\
\hline 0 & -26.08362 & 0 \\
1 & -17.38859 & -0.7937005 \\
2 & -11.59129 & -1.433775 \\
3 & -7.725046 & -2.249475 \\
4 & -5.144445 & -3.417035 \\
5 & -3.417035 & -5.144445 \\
6 & -2.249475 & -7.725045 \\
7 & -1.433776 & -17.38859 \\
8 & -0.7937006 & -11.59129 \\
9 & $-7.7054578 E-08$ & -17.38859 \\
10 & - & -26.08362 \\
\hline
\end{tabular}

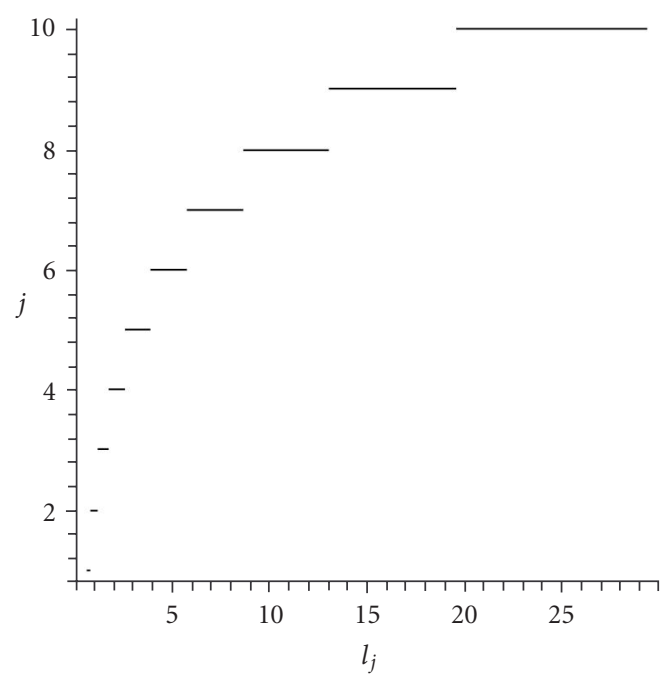

Figure 5.2. The successive gaps of the sequence generated by formula (5.7).

5.2. Modification of Newton algorithm and global convergence. Notice that the algorithm

$$
x_{+}=x+\frac{1-x^{3}}{3 x^{2}}
$$

converges only for any $x$ close of the root, otherwise one does not have a global convergence. For that we can interpret the quantity $\left(1-x^{3}\right) / 3 x^{2}$ as a direction, the long of which we do a displacement $\lambda$ to force the global convergence. A new algorithm is proposed therefore

$$
x_{+}=\mathcal{N}(x, \lambda)=x+\lambda \frac{1-x^{3}}{3 x^{2}}, \quad \lambda>0 .
$$




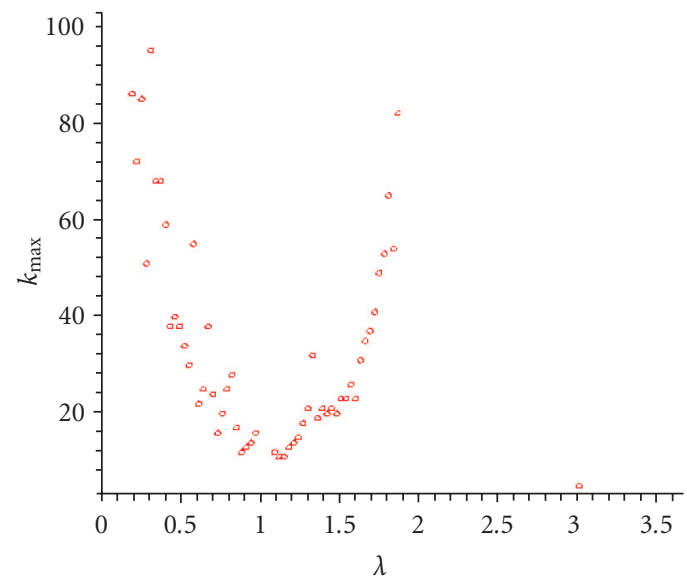

Figure 5.3. Modified Newton algorithm with different values of $\lambda$ for a chosen $x=-3.417034624$.

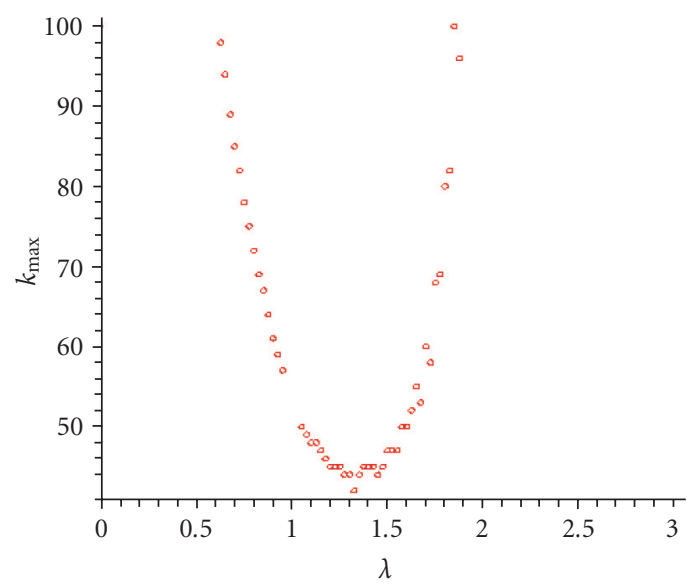

Figure 5.4. Modified Newton algorithm with different values of $\lambda$ for $x=2.88418640 \times 10^{8}$.

The numerical tests (Figures 5.3, 5.4 and 5.5) prove that for some values of $\lambda$; we can give back a no-converging point (which belongs to Julia set) in a convergent point (then in Fatou set).

Besides, the bifurcation diagram (see Figure 5.6) permits us to affirm that in the interval ] 0,2] there is convergence, on the other hand in the interval ]2,7] there is divergence of cyclic type due to a succession of period doubling and apparition of chaos.

5.3. The two-dimensional map. It is easy to calculate the three roots of (1.3):

$$
z_{k}=e^{(2 / 3)(k-1) \pi i}, \quad k=1,2,3,
$$




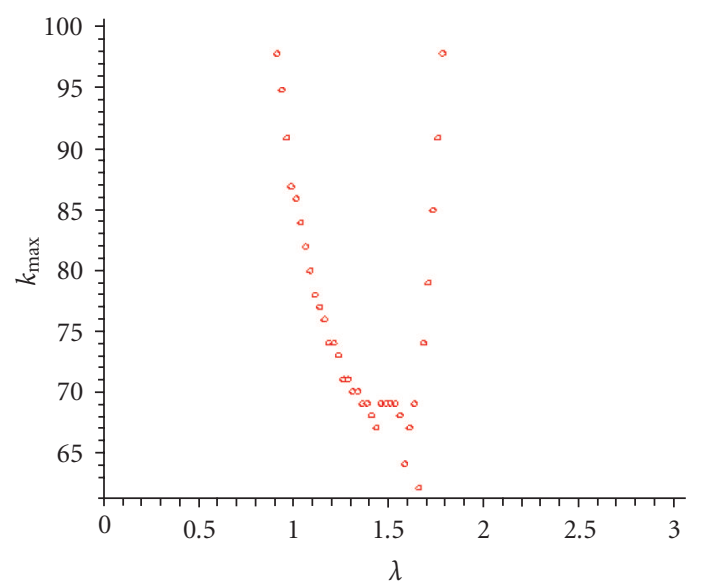

Figure 5.5. Modified Newton algorithm for different values of $\lambda$ with $x=-2.88418640 \times 10^{-8}$.

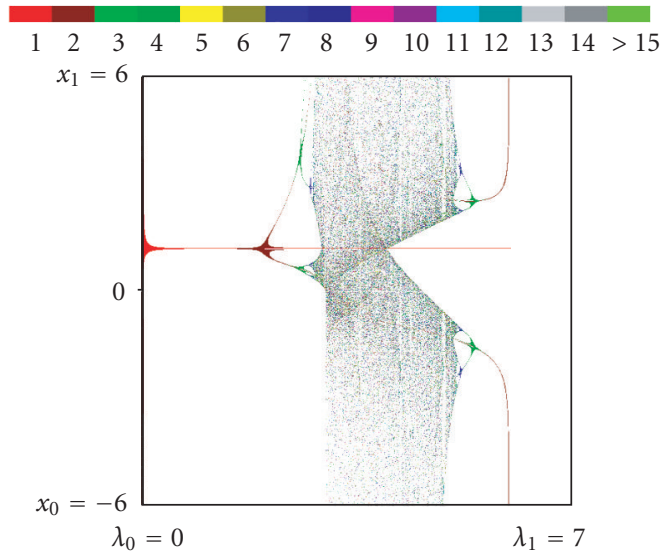

Figure 5.6. Bifurcation diagram of the $\mathcal{N}(x, \lambda)$ algorithm.

then

$$
z_{1}=1, \quad z_{2,3}=-\frac{1}{2} \pm i \frac{1}{2} \sqrt{3}
$$

So one can consider couples $(1,0),(-1 / 2,(1 / 2) \sqrt{3}),(-1 / 2,-(1 / 2) \sqrt{3})$ as solutions of the nonlinear system

$$
f(x, y)=\left(\begin{array}{l}
f_{1}(x, y) \\
f_{2}(x, y)
\end{array}\right)=\left(\begin{array}{c}
x^{3}-3 x y^{2}-1 \\
3 x^{2} y-y^{3}
\end{array}\right)=0
$$

obtained by considering $z=x+i y$ and $f(z)=f_{1}(x, y)+i f_{2}(x, y)$. Let us pass now to the crucial construction of $f$ schematized in Figure 5.7. 


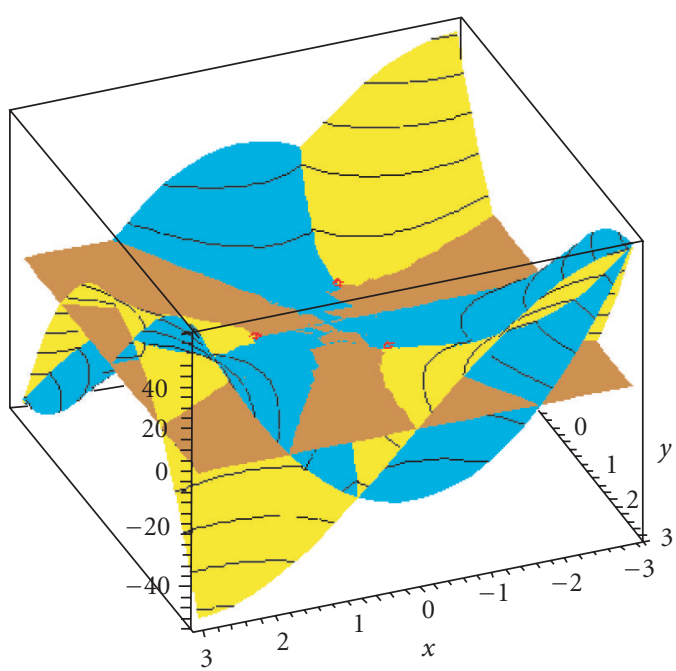

Figure 5.7. Solutions of the cubic equation.

Apply now to the map (5.17) the Newton algorithm by the following form

$$
X_{+}=X-J^{-1}(X) f(X)
$$

where $X=(x, y)$, and $J^{-1}(X)$ is the Jacobian inverse $f$ evaluated in $X$.

Such that the Jacobian is given by

$$
J(x, y)=\left(\begin{array}{cc}
3 x^{2}-3 y^{2} & -6 x y \\
6 x y & 3 x^{2}-3 y^{2}
\end{array}\right)
$$

which is invertible, the algorithm (5.18) is well defined and converges locally to the solution of (5.17), its eigenvalues are then

$$
\lambda_{1}=3(x+i y)^{2}, \quad \lambda_{2}=3(x-i y)^{2},
$$

then $J(x, y)$ is singular for $(x, y)=(0,0)$, elsewhere it does not have a definite sign and its inverse is expressed by

$$
J^{-1}(x, y)=\frac{1}{9\left(x^{2}+y^{2}\right)^{2}}\left(\begin{array}{cc}
3 x^{2}-3 y^{2} & 6 x y \\
-6 x y & 3 x^{2}-3 y^{2}
\end{array}\right) .
$$




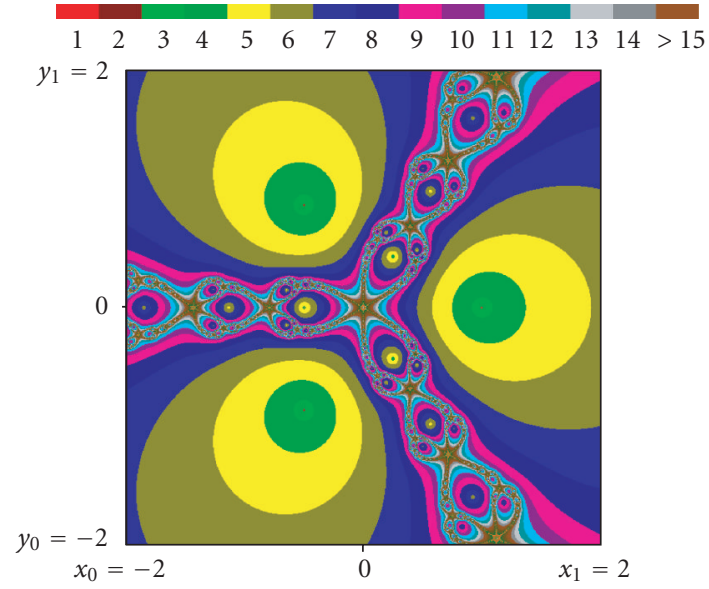

Figure 5.8. The figure shows the basins of attraction for Newton's method applied to the equation $z^{3}-1=0$.

If we replace in (5.18) we obtain the formula

$$
\begin{gathered}
x_{+}=\frac{2 x^{5}+4 x^{3} y^{2}+2 x y^{4}+x^{2}-y^{2}}{3\left(x^{2}+y^{2}\right)^{2}}, \\
y_{+}=\frac{2 y\left[\left(x^{2}+y^{2}\right)^{2}-x\right]}{3\left(x^{2}+y^{2}\right)^{2}} .
\end{gathered}
$$

Remarks 5.1. We show three sets $A_{1}, A_{2}, A_{3}$ and their boundaries, such that $A_{i}=$ $\left\{z ; f^{n}(z) \rightarrow z_{i}\right\}$ with some properties (see Figure 5.8).

(1) Symmetry: if you rotate the $z$-plane by $120^{\circ}$, then $A_{1}$ becomes $A_{2}, A_{2}$ becomes $A_{3}$, and $A_{3}$ becomes $A_{1}$.

(2) The closure of $A_{1} \cup A_{2} \cup A_{3}$ is equal to the whole $z$-plane. Thus any point $z$ is either in $A_{1}, A_{2}, A_{3}$ or their boundaries.

(3) The boundaries of $A_{1}, A_{2}$, and $A_{3}$ are the same and very exotic. If we denote $\partial A=$ boundary of the set $A$, then $\partial A_{1} \cup \partial A_{2} \cup \partial A_{3}$ is called the Julia set and denote it as $\mathscr{F}(\mathcal{N})$.

(4) 0 and its preimages are in $\mathscr{S}(\mathcal{N})$. Notice that $f(0)=\infty$. Hence, 0 is the hole leading to infinity. Clearly $0 \notin \partial A_{1} \cup \partial A_{2} \cup \partial A_{3}$. Thus $0 \in \mathscr{J}(\mathcal{N})$. We then conclude that all the preimages of 0 are also in $\mathscr{S}(\mathcal{N})$. Note that these preimages of 0 are countable.

(5) Other aperiodic points which wander about in $\mathscr{S}(\mathcal{N})$ forever must also be in $\mathscr{S}(\mathcal{N})$. These points are uncountable and make up the majority of $\mathscr{G}(\mathcal{N})$.

(6) There is a dense orbit in $\mathscr{L}(\mathcal{N})$, the map (5.3) is chaotic on $\mathscr{L}(\mathcal{N})$.

It is clear the first element of the Julia set $\mathscr{F}(\mathcal{N})$ relative to the map (5.3) is the point $(0,0)$, we can obtain the others elements by solving with regard to $z$ the equation of 
Newton

$$
2 z^{3}-3 z_{+} z^{2}+1=0
$$

This last admits three complex solutions given by

$$
\begin{aligned}
& z_{1}=\frac{1}{2}\left[\varphi\left(z_{+}\right)+z_{+}\right], \\
& z_{2}=\frac{1}{4}\left[-\varphi\left(z_{+}\right)+2 z_{+}+I \sqrt{3} \psi\left(z_{+}\right)\right], \\
& z_{3}=\frac{1}{4}\left[-\varphi\left(z_{+}\right)+2 z-I \sqrt{3} \psi\left(z_{+}\right)\right],
\end{aligned}
$$

where

$$
\begin{aligned}
& \varphi(z)=\sqrt[3]{-2+z^{3}+2 \sqrt{1-z^{3}}}+\frac{z^{2}}{\sqrt[3]{-2+z^{3}+2 \sqrt{1-z^{3}}}}, \\
& \psi(z)=\sqrt[3]{-2+z^{3}+2 \sqrt{1-z^{3}}}-\frac{z^{2}}{\sqrt[3]{-2+z^{3}+2 \sqrt{1-z^{3}}}} .
\end{aligned}
$$

While projecting on real and imaginary axes, we have the following formulas in $\mathbb{R}^{2}$ :

$$
\begin{aligned}
& x_{1}=\frac{1}{2}\left[\eta+x_{+}+\frac{2 x_{+} y_{+} \eta-\beta \xi}{\alpha}\right], \\
& y_{1}=\frac{1}{2}\left[\eta+y_{+}+\frac{\beta \eta+2 x_{+} y_{+} \xi}{\alpha}\right], \\
& x_{2}=\frac{1}{4 \alpha}\left[\alpha\left(2 x_{+}-\xi-\sqrt{3} \eta\right)+\beta(\xi+\sqrt{3} \eta)+2 x_{+} y_{+}(\sqrt{3} \xi-\eta)\right], \\
& y_{2}=\frac{1}{4 \alpha}\left[\alpha\left(2 y_{+}-\eta+\sqrt{3} \xi\right)+\beta(\sqrt{3} \xi-\eta)-2 x_{+} y_{+}(\sqrt{3} \eta+\xi)\right], \\
& x_{3}=\frac{1}{4 \alpha}\left[\alpha\left(2 x_{+}-\xi+\sqrt{3} \eta\right)+\beta(\xi-\sqrt{3} \eta)-2 x_{+} y_{+}(\sqrt{3} \xi+\eta)\right], \\
& y_{3}=\frac{1}{4 \alpha}\left[\alpha\left(2 y_{+}-\eta-\sqrt{3} \xi\right)-\beta(\sqrt{3} \xi+\eta)+2 x_{+} y_{+}(\sqrt{3} \eta-\xi)\right],
\end{aligned}
$$

with

$$
\begin{aligned}
\varphi\left(z_{+}\right) & =\xi+I \eta, \\
\alpha & =\xi^{2}+\eta^{2}, \\
\beta & =y_{+}^{2}-x_{+}^{2} .
\end{aligned}
$$

In Table 5.3, we remark the behavior of the Newton algorithm with an initial condition in the Julia set (case of nonconvergence) whereas for a neighboring point the qualitative behavior changes.

We now show how to construct the Julia set with the help of the inverse Newton algorithm defined by formulas (5.28), (5.29), and (5.30). 
14 Fractal Newton basins

Table 5.3. Cases of convergence and of nonconvergence for Newton algorithm.

\begin{tabular}{lc}
\hline$x_{0}$ & $k_{\max }$ \\
\hline$(-5.1444445,0)$ & - \\
$(-5.1444,0)$ & 59 \\
$(3.862522716,-6.690085583)$ & - \\
$(3.8625,-6.6900)$ & 59 \\
$(29.34438653,-50.82596840)$ & - \\
$(29.344,-50.825)$ & 61 \\
$(8.694293987,15.05895892)$ & - \\
$(8.6942,15.0589)$ & 69 \\
\hline
\end{tabular}

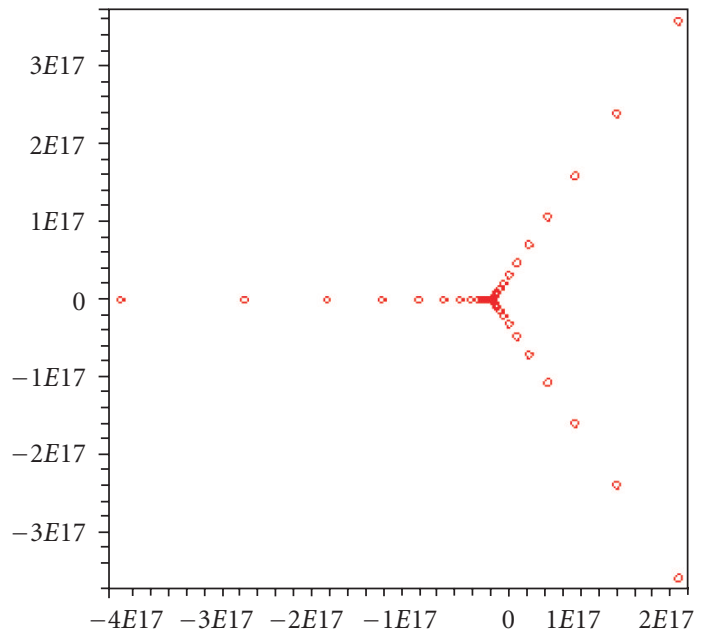

Figure 6.1. First generation of Julia set.

\section{Julia set by the inverse algorithm}

Let $T_{1}, T_{2}, T_{3}$ be three maps (5.28), (5.29), and (5.30) such that

$$
\begin{aligned}
& \left(x_{1}, y_{1}\right)=T_{1}(x, y), \\
& \left(x_{2}, y_{2}\right)=T_{2}(x, y), \\
& \left(x_{3}, y_{3}\right)=T_{3}(x, y) .
\end{aligned}
$$

We define the first generation of Julia set by

$$
\mathscr{D}_{1}=\left\{T_{1}^{j}(0,0), T_{2}^{j}(0,0), T_{3}^{j}(0,0), j=0,1,2,3, \ldots\right\},
$$

it is clear that the set $\mathscr{D}_{1}$ is included in the Julia set $\mathscr{I}(\mathcal{N})$ (see Figure 6.1). 


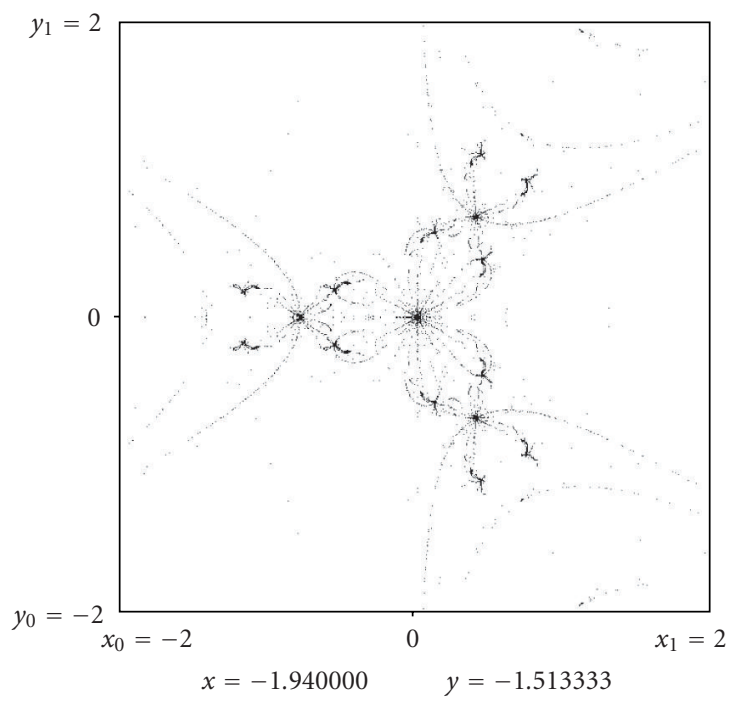

Figure 6.2. Second generation of Julia set.

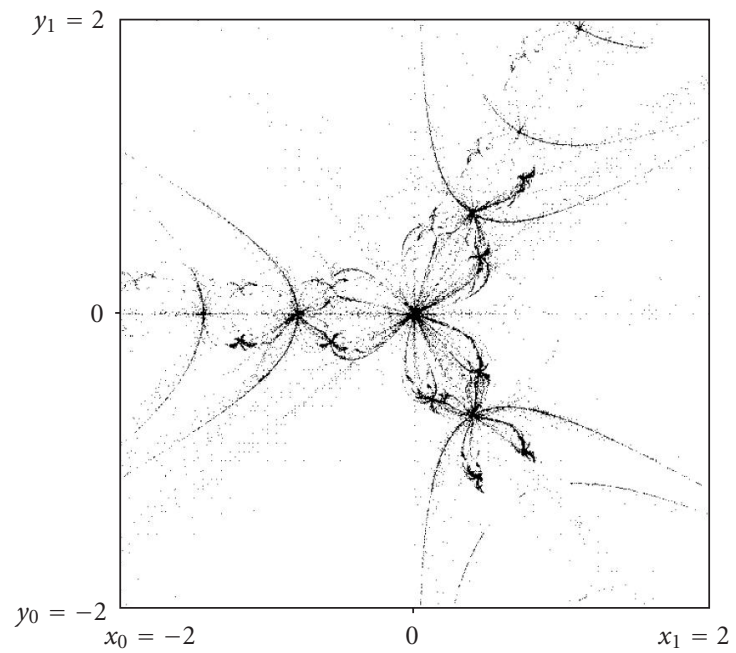

Figure 6.3. Third generation of Julia set.

Since every point of $\mathscr{E}(\mathcal{N})$ admits three preimages by $T_{1}, T_{2}, T_{3}$; we can define so the second generation of Julia set is defined by

$$
\mathscr{D}_{2}=\left\{T_{1}^{j}(u), T_{2}^{j}(u), T_{2}^{j}(u) ; u \in \mathscr{D}_{1}, j=0,1,2, \ldots\right\}
$$


and thus, the Julia set of $k$ th generation is given by

$$
\mathscr{D}_{k}=\left\{T_{1}^{j}(u), T_{2}^{j}(u), T_{3}^{j}(u) ; u \in \mathscr{D}_{k-1}, j=0,1,2,3, \ldots\right\} .
$$

It is obvious if we consider $\mathscr{D}_{0}=\{(0,0)\}$, we have then a relation in this following scheme

$$
\mathscr{D}_{0} \subset \mathscr{D}_{1} \subset \mathscr{D}_{2} \subset \cdots \subset \mathscr{D}_{k} \subset \cdots
$$

Since the point $(0,0)$ is not a fixed point for the three applications $T_{1}, T_{2}, T_{3}$ (see Figures $6.2,6.3)$, then the Julia set $\mathscr{F}(\mathcal{N})$ is well determined by (5.28), (5.29), and (5.30).

Proposition 6.1. Let $\left(\mathscr{D}_{k}\right)_{k=1,2, \ldots}$, the sets defined previously; then

$$
\mathscr{D}_{\infty}=\mathscr{F}(\mathcal{N}) \text {. }
$$

An immediate and important consequence is that the Fatou set $\mathscr{F}(\mathcal{N})$ (the set of convergent points) is the complementary in $\overline{\mathbb{C}}$ of $\mathscr{D}_{\infty}$, and then

$$
\mathscr{F}(\mathcal{N})=\overline{\mathbb{C}} \backslash \mathscr{D}_{\infty}
$$

\section{References}

[1] M. S. Bazara, H. D. Sherali, and C. M. Shetty, Nonlinear Programming: Theory and Algorithms, John Wiley \& Sons, New York, 1993.

[2] A. Cayley, Desiderata and suggestions: no. 3.the Newton-Fourier imaginary problem, American Journal of Mathematics 2 (1879), no. 1, 97.

[3] J. Milnor, Remarks on iterated cubic maps, Experimental Mathematics 1 (1992), no. 1, 5-24.

[4] _ Dynamics in One Complex Variable. Introductory Lectures, 2nd ed., Vieweg, Wiesbaden, 2000.

M. L. Sahari: Centre Universitaire d'El-Tarf, 36000 El Tarf, Algeria

E-mail address: mlsahari@yahoo.fr

I. Djellit: Department of Mathematics, University of Annaba, BP 12, 23000 Annaba, Algeria

E-mail address: a_djellit@hotmail.com 


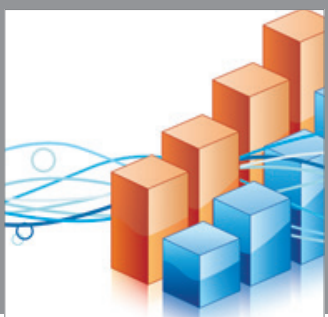

Advances in

Operations Research

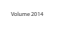

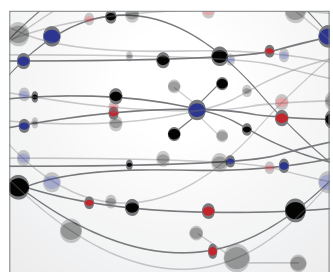

\section{The Scientific} World Journal
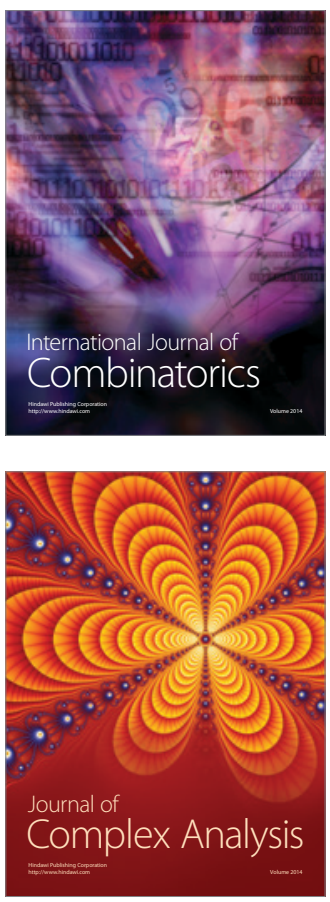

International Journal of

Mathematics and

Mathematical

Sciences
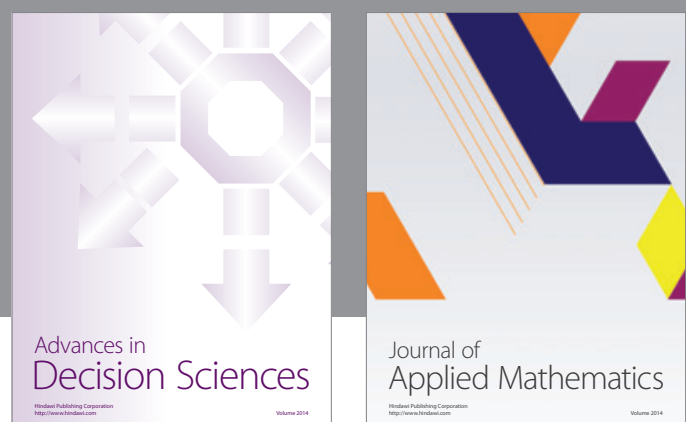

Journal of

Applied Mathematics
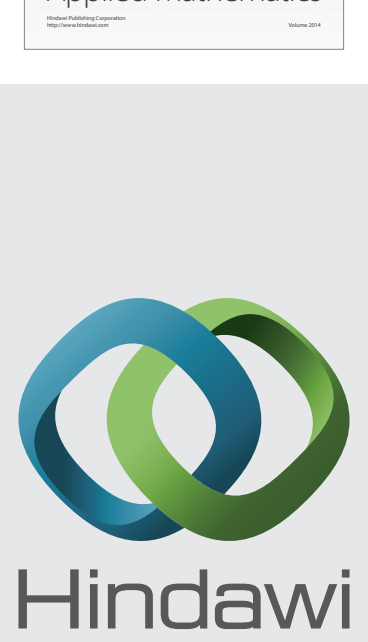

Submit your manuscripts at http://www.hindawi.com
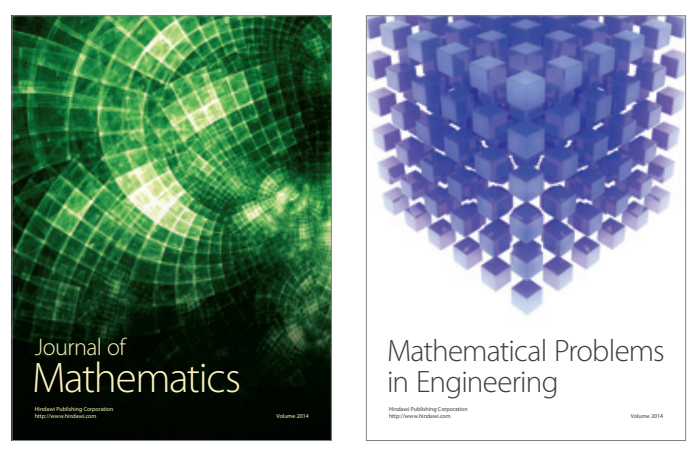

Mathematical Problems in Engineering
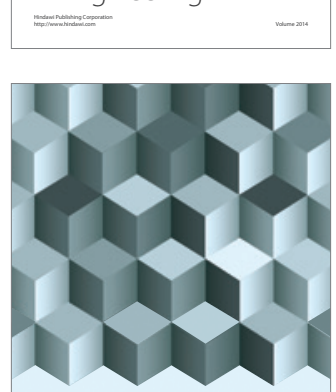

Journal of

Function Spaces
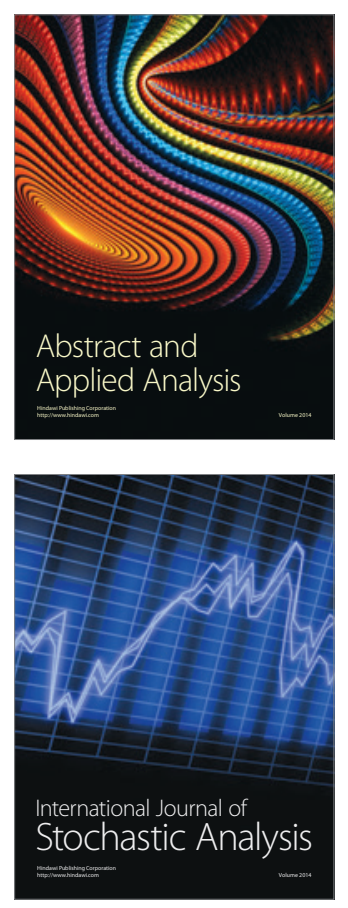

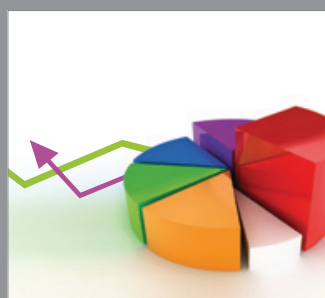

ournal of

Probability and Statistics

Promensencen
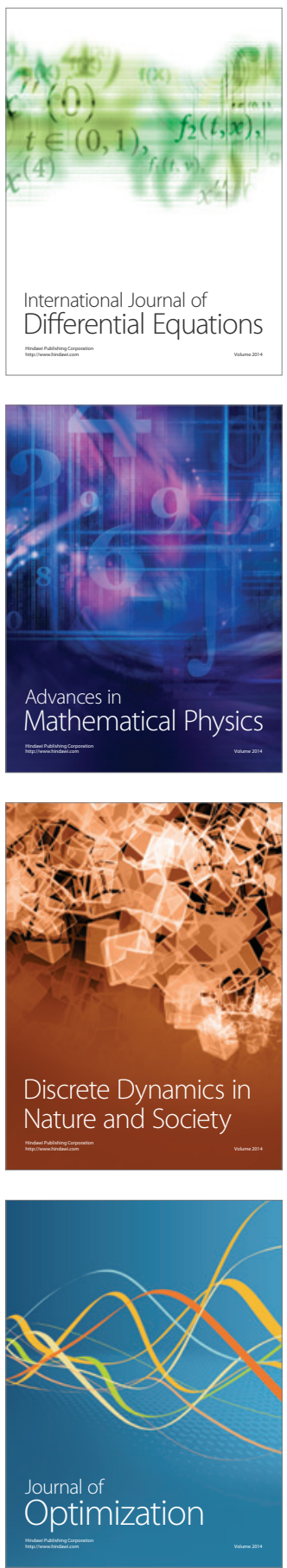